\title{
SDGs and Cartography, a conceptual connection
}

\author{
Lemmens, R.L.G. ${ }^{\mathrm{a}, *}$, and Kraak, M.J. ${ }^{\mathrm{b}}$ \\ ${ }^{a}$ University of Twente,r.l.g.lemmens@utwente.nl \\ ${ }^{b}$ University of Twente, m.j.kraak@utwente.nl \\ * Corresponding author
}

Keywords: SDG, cartography, concept map, living textbook

\begin{abstract}
:
In partnership with the United Nations Geospatial Information Section the International Cartographic Association (ICA) has recently published the textbook "Mapping for a Sustainable World". It aims to bring best practices and methods in cartography to support to uncover challenges and achievements towards the Sustainable Development Goals (SDGs).

The maps and diagrams discussed help to better understand the relationship between humans and their environments as well as enable us to monitor SDG indicators and communicate their uneven global footprints. The objective of the maps and diagrams is to support decision making by local and national authorities, as well as to promote public awareness of global issues to encourage these authorities to act. The book discusses and explains why some design decisions are not suitable for certain mapping contexts, which results in flawed or even misleading maps and diagrams. However, issues also arise from improper data handling, distracting symbols and text, confusing map elements, and the (mis)use of software defaults. The main objective is not to offer fixed rules, but to hand recommendations to avoid common pitfalls in cartographic design.
\end{abstract}

The book [1] is published as an open access document according to the CC BY-NC license [2] and is available via the United Nations Digital Library. The indicator data and boundaries will also be made available. This open content is part of an open education environment. This environment will also include other open educational resources. This paper describes one of these tools that will help both teachers and students structure there teaching and learning respectively in the domain of cartography with a strong link to the Sustainable Development Goals.

With the ubiquitous availability of online resources and media, the process of teaching and learning, and knowledge sharing in general, has evolved significantly over the last decades. With the growing demand for flexibility in education, the process of learning has become increasingly non-linear; knowledge acquisition is done via different pathways over several content resources and teaching components. At the same time, increasingly use is made of a combination of independently created knowledge resources and the meaning of the connections between them become obscure. In such cases a clear structure of content is needed to understand the conceptual connections between the different knowledge components.

In the context of ITC's Open Education environment, a tool was developed called the Living Textbook [3] to create and consume both knowledge content and structure. The latter is represented in a concept map, which holds small units of content, represented as circles, and their relationships, represented as text-labelled arrows. Each combination of two concepts forms a so-called triple, a short sentence that describes their relationship, e.g. Choropleth map is a kind of Thematic map.

Use in education. The core content of the education at ITC has been migrated from a traditional textbook into the Living Textbook. This allows students to set their own learning paths and supports us to connect to content of follow-up courses in a constructive manner. In other education activities the Living Textbook is used by students to create their own content, based on supervised research activities.

Use in knowledge sharing. In several projects we use the Living Textbook to collaboratively create a knowledge base of shared content. In the EO4GEO project [4] the Living Textbook is the main editor for the creation of the Body of Knowledge (BoK) for Geo-information and Earth Observation. This BoK contains over 900 connected concepts created and maintained by a group of experts. The concepts form the building blocks for linking education and the labour market in the European EO/GI sector. In another project we use the same method to build a consolidated view on Citizen Science and related project activities. 


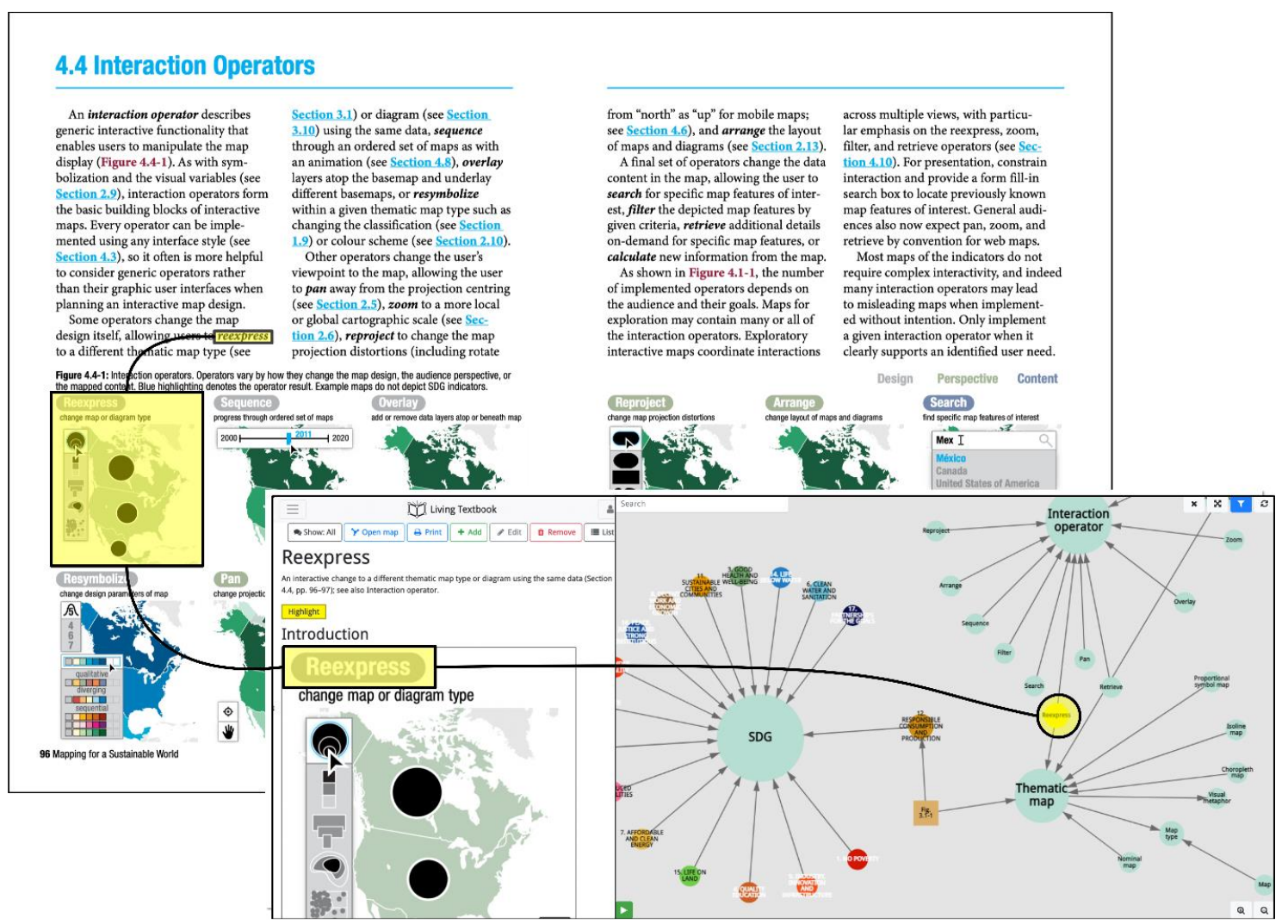

Figure 1. Implementation of an example of a connection between the book 'Mapping for a Sustainable World' and the concept map in the Living Textbook

In this paper we are following a similar approach (see Figure 1) to connect concepts in cartography and to make explicit how they can be applied to SDG issues, as described in the book. In all of the conceptual modelling done with concept maps, one of the challenges is to find the right granularity of concepts and comprehensive relationships between the concepts, which are understandable for end users. In our case these are researchers and educators who would like to reuse specific content parts of the book and refer to it through hyperlinks. We have chosen to take the glossary of the textbook as a starting point and started to conceptualise each chapter as well as the SDG goals, targets and indicators. In this way a hierarchical structure is prevalent in cartographic concepts, supporting an intuitive way of exploration, in combination with specific relations between SDGs, map representations and cartographic concepts.

Research and development is ongoing to improve several aspects of the use of concept maps, through 1) learning analytics, 2) alternative concept map visualisations and 3) application of semantic web principles and linked open data, see [5]. A link is foreseen with ICA's BoK which is currently being developed.

[1] Kraak MJ, RE Roth, B Ricker, A Kagawa, and G Le Sourd. 2020. Mapping for a Sustainable World. United Nations: New York, NY (USA). (https://digitallibrary.un.org/record/3898826)

[2] http://creativecommons.org/licences/by/3.0/igo/

[3] https://www.itc.nl/about-itc/organization/resources-facilities/living-textbook/

[4] http://www.eo4geo.eu/bok/

[5] https://www.itc.nl/bodiesofknowledge 